Pacific

Journal of

Mathematics

EXPLOSIONS NEAR ISOLATED UNSTABLE ATTRACTORS

Konstantin Athanassopoulos

Volume $210 \quad$ No. 2

June 2003 


\title{
EXPLOSIONS NEAR ISOLATED UNSTABLE ATTRACTORS
}

\author{
Konstantin Athanassopoulos
}

\begin{abstract}
We describe the set of explosive orbits in the region of attraction of an unstable attractor which is isolated in the sense of C.C. Conley. Sufficient conditions are given for the existence of explosions in certain parts of the region of attraction and for an unstable attractor to have finitely generated integral Alexander-Spanier cohomology groups. Finally, we study the case of singularities that are unstable attractors in flows on the 2-sphere.
\end{abstract}

\section{Introduction.}

One of the most studied parts of the phase space of a continuous flow on a separable, locally compact, metrizable space $M$ is the region of attraction $W$ of an asymptotically stable compact invariant set $A$, that is a Lyapunov stable attractor. It is well-known (see [2, Section 10], [3, Theorem V.2.9], [8]) that there exists a strictly decreasing along the orbits in $W \backslash A$ uniformly unbounded Lyapunov function $f: W \rightarrow \mathbb{R}^{+}$with $f^{-1}(0)=A$. For any $c>0$, the level set $f^{-1}(c)$ is a compact global section to the restricted flow in $W \backslash A$, which is therefore parallelizable. If $M$ is a locally compact ANR, $A$ has the shape of the compact ANR $f^{-1}([0, c])$, which has the homotopy type of a compact polyhedron, and which is a strong deformation retract of $W$. Thus, $A$ and $W$ have isomorphic and finitely generated Alexander-Spanier cohomology (see [9]).

On the other hand, not much is known about the structure of the flow in the region of attraction of an unstable attractor. In this case there might be orbits in $W \backslash A$ whose positive prolongational limit set is not contained in $A$, and so the flow explodes at these orbits. In general we say that the flow expodes at an orbit if the positive limit set of the orbit is not equal to its positive prolongational limit set.

This work has two parts. In the first part we study the explosions of the flow in the region of attraction of an unstable attractor, under the additional assumption that it is an isolated invariant set in the sense of C.C. Conley. First we show in Theorem 3.4 that if $A$ is an invariant continuum and an isolated unstable attractor such that $W \backslash A$ is connected, then there exist explosions in $W \backslash D^{+}(A)$ or $D^{+}(A)=M$, where $D^{+}(A)$ is the positive first 
prolongation of $A$. The set of orbits in $W \backslash A$ at which the flow explodes is completely determined by a suitable compact subset of the boundary of an isolating block of $A$.

The positive first prolongation $D^{+}(A)$ of an unstable attractor $A$ is an asymptotically stable compact invariant set with the same region of attraction as $A$ (see $[\mathbf{2}$, Theorem 8.20]). Simple examples show that the flow may or may not explode at some orbit in $D^{+}(A) \backslash A$. In Theorem 3.5 we give a sufficient topological condition on the region of attraction in order the flow to explode at some orbit in $D^{+}(A) \backslash A$. We derive also some topological properties of a connected isolated unstable attractor in case there is essentially no explosion in $D^{+}(A) \backslash A$ and $D^{+}(A) \backslash A$ is topologically not too bad. More precisely, we prove in Theorem 3.7 that if $D^{+}(A) \backslash A$ is an ANR and the first positive prolongational limit set of every point in $D^{+}(A) \backslash A$ is contained in $A$ then the integral Alexander-Spanier cohomology groups of $A$ are finitely generated. The assumption that $D^{+}(A) \backslash A$ is an ANR is of course resrtictive in general.

In the second part we study the case where $A$ is a fixed point of a flow on a 2-manifold $M$, which is an isolated unstable attractor. In this case the set of orbits in $W \backslash A$ at which the flow explodes is finite, as we prove in Corollary 4.3. If $M$ is the 2 -sphere, then we prove in Theorem 4.5 that $D^{+}(A) \backslash A$ has finitely many connected components and each one of them is either an orbit homeomorphic to $\mathbb{R}$ or a noncompact 2-manifold with nonempty and noncompact boundary, and with finitely many boundary components. Hence $D^{+}(A) \backslash A$ is an ANR. Moreover, as we prove in Theorem 4.7 the restricted flow in $D^{+}(A) \backslash A$ is completely unstable. These are not local results, as global properties of the flow have to be taken into account.

\section{Explosions and isolated invariant sets.}

Let $\phi$ be a continuous flow on a separable, locally compact, metrizable space $M$. We shall denote by $\phi(t, x)=t x$ the translation of the point $x \in M$ along its orbit in time $t \in \mathbb{R}$. We shall also write $\phi(I \times A)=I A$, for $I \subset \mathbb{R}$ and $A \subset M$. The orbit of $x$ will be denoted by $C(x)$, its positive semiorbit by $C^{+}(x)$ and the negative by $C^{-}(x)$. The positive limit set of $x \in M$ is the closed, invariant set

$$
L^{+}(x)=\left\{y \in M: t_{n} x \rightarrow y \text { for some } t_{n} \rightarrow+\infty\right\}
$$

and describes the behavior at infinity of $C^{+}(x)$. The behavior of the flow at infinity near the point $x \in M$ is described through its (first) positive prolongational limit set

$$
J^{+}(x)=\left\{y \in M: t_{n} x_{n} \rightarrow y \text { for some } x_{n} \rightarrow x \text { and } t_{n} \rightarrow+\infty\right\}
$$

which is also closed and invariant. The positive first prolongation of $x$ is the positively invariant closed set $D^{+}(x)=C^{+}(x) \cup J^{+}(x)$. The negative 
versions are defined by reversing time. Obviously, $L^{+}(x) \subset J^{+}(x)$. We say that the flow explodes at the orbit of the point $x \in M$ in positive time if $J^{+}(x) \neq L^{+}(x)$.

A compact invariant set $A \subset M$ is called isolated if it has a compact neighbourhood $V$ such that $A$ is the maximal invariant set in $V$. Each such $V$ is called an isolating neighbourhood of $A$. It is known (see [4], [5]) that every isolating neighbourhood of $A$ contains a smaller isolating neighbourhood $N$ of $A$ such that there are compact sets $N^{+}, N^{-} \subset \partial N$ with the following properties:

(i) $\partial N=N^{+} \cup N^{-}$.

(ii) For every $x \in N^{+}$there exists $\epsilon>0$ such that $[-\epsilon, 0) x \subset M \backslash N$ and for every $y \in N^{-}$there exists $\delta>0$ such that $(0, \delta] y \subset M \backslash N$.

(iii) For every $x \in \partial N \backslash N^{+}$there exists $\epsilon>0$ such that $[-\epsilon, 0) x \subset \operatorname{int} N$ and for every $y \in \partial N \backslash N^{-}$there exists $\delta>0$ such that $(0, \delta] y \subset \operatorname{int} N$.

The triad $\left(N, N^{+}, N^{-}\right)$is called an isolating block of $A$. The set $N^{+}$is the entrance set and $N^{-}$is the exit set of the isolating block. The sets $A^{ \pm}=$ $\left\{x \in N: C^{ \pm}(x) \subset N\right\}$ and $\alpha^{ \pm}=\partial N \cap A^{ \pm}$are compact and $A=A^{+} \cap A^{-}$. Moreover, $\varnothing \neq L^{+}(x) \subset A$ for every $x \in A^{+}$and $\alpha^{+} \subset N^{+} \backslash N^{-}$.

If $M$ is a smooth $n$-manifold and the flow is smooth, then every neighbourhood of an isolated invariant set $A$ contains a smooth isolating block $\left(N, N^{+}, N^{-}\right)$of $A$. This means that $N$ is a smooth compact $n$-manifold with boundary $\partial N=N^{+} \cup N^{-}$, the sets $N^{+}$and $N^{-}$are smooth compact $(n-1)$-manifolds with common boundary $N^{+} \cap N^{-}$, which is a smooth compact $(n-2)$-manifold (without boundary) and on which the flow is externally tangent to $N$. Moreover, the flow is transverse to $N^{+} \backslash N^{-}$into $N$ and transverse to $N^{-} \backslash N^{+}$out of $N$ (see [6]).

Lemma 2.1. Let $A$ be an isolated invariant set in $M$ and $\left(N, N^{+}, N^{-}\right)$be an isolating block of $A$.

(a) If $x \in \operatorname{int}_{\partial N} \alpha^{+}$, then $\varnothing \neq J^{+}(x) \subset A$.

(b) If $x \in \partial_{\partial N} \alpha^{+}$, then $J^{+}(x) \cap \alpha^{-} \neq \varnothing$.

Proof. (a) Let $W$ be an open neighbourhood of $x$ in $M$ such that $W \cap \partial N \subset$ $\alpha^{+}$. Let $y \in J^{+}(x)$ and suppose that $y \notin A$. There are open sets $U$ and $Y$ in $M$ such that $A \subset U \subset \operatorname{int} N, y \in Y$ and $\bar{U} \cap \bar{Y}=\varnothing$. Since $\varnothing \neq L^{+}(x) \subset A$, there exists $t>0$ such that $C^{+}(t x) \subset U$. There are $t_{n}>t$, $x_{n} \in W \cap \partial N, n \in \mathbb{N}$, such that $t_{n} \rightarrow+\infty, x_{n} \rightarrow x$ and $t_{n} x_{n} \rightarrow y$. So, there are $s_{n}>t_{n}$ such that $s_{n} x_{n} \in \partial U, n \in \mathbb{N}$. By compactness, the sequence $\left\{s_{n} x_{n}: n \in \mathbb{N}\right\}$ has a limit point $z \in \partial U$. For every $s \in \mathbb{R}$ we have eventually $\left(s+s_{n}\right) x_{n} \in C^{+}\left(x_{n}\right)$. Therefore, $s z \in N$ for every $s \in \mathbb{R}$. This contradicts our hypothesis that $A$ is isolated.

(b) There is a sequence $\left\{x_{n}: n \in \mathbb{N}\right\}$ of points in $N^{+} \backslash \alpha^{+}$converging to $x$. Let $\sigma^{+}\left(x_{n}\right)$ be the time $C^{+}\left(x_{n}\right)$ exits $N$. Then $\sigma^{+}\left(x_{n}\right) x_{n} \in N^{-} \backslash \alpha^{-}$and 
$\sigma^{+}\left(x_{n}\right) \rightarrow+\infty$, because $x \in \alpha^{+}$. By compactness, the sequence $\left\{\sigma^{+}\left(x_{n}\right) x_{n}\right.$ : $n \in \mathbb{N}\}$ has a limit point $y \in J^{-}(x) \cap N^{-}$. For each $s<0$ we have eventually $0<\sigma^{+}\left(x_{n}\right)+s<\sigma^{+}\left(x_{n}\right)$ and therefore $\left(\sigma^{+}\left(x_{n}\right)+s\right) x_{n} \in N$. It follows that sy $\in N$ for every $s<0$, that is $y \in J^{+}(x) \cap \alpha^{-}$.

Remark 2.2. Let $A$ be an isolated invariant set and let $x \in M$. Let $\left(N_{i}, N_{i}^{+}, N_{i}^{-}\right), i=1,2$, be two isolating blocks of $A$ such that $N_{1} \subset N_{2}$. Then, $J^{+}(x) \cap \alpha_{2}^{-}$is homeomorphic to $J^{+}(x) \cap \alpha_{1}^{-}$. Indeed, for every $x \in \alpha_{2}^{-}$ there is a unique $\tau(x) \leq 0$ such that $\tau(x) x \in \alpha_{1}^{-}$. It is easy to see that the function $\tau: \alpha_{2}^{-} \rightarrow \mathbb{R}$ is continuous. Define now the continuous injection $h: \alpha_{2}^{-} \rightarrow \alpha_{1}^{-}$by $h(x)=\tau(x) x$. This is also onto, because for every $z \in \alpha_{1}^{-}$ we have $C^{+}(z) \nsubseteq N_{2}$. Thus $h$ is a homeomorphism, by compactness. It is now obvious that $h\left(J^{+}(x) \cap \alpha_{2}^{-}\right)=J^{+}(x) \cap \alpha_{1}^{-}$. If we have two isolating blocks of the isolated invariant set $A$, then we can always find a smaller isolating block. So, the homeomorphism type of $J^{+}(x) \cap \alpha^{-}$does not depend on the choice of the isolating block. If $x \in M$ is such that $\varnothing \neq L^{+}(x) \subset A$, it is contained in $J^{+}(x) \backslash L^{+}(x)$ and its size and topological complexity may give information on the explosion of the flow at $x$. However, it seems to be difficult to deal with the set $J^{+}(x) \cap \alpha^{-}$.

\section{Isolated unstable attractors.}

Let again $\phi$ be a continuous flow on a separable, locally compact, metrizable space $M$ and $A \subset M$ be a compact invariant set. The invariant set

$$
W^{+}(A)=\left\{x \in M: \varnothing \neq L^{+}(x) \subset A\right\}
$$

is the region of attraction (or stable manifold) of $A$. If $W^{+}(A)$ is an open neighbourhood of $A$, then $A$ is called an attractor.

A compact invariant set $A \subset M$ is called stable (in the sense of Lyapunov) if every neighbourhood of $A$ contains a positively invariant neighbourhood of $A$. This is equivalent to saying that $\varnothing \neq J^{+}(x) \subset A$ for every $x \in A$ (see [3, Theorem 1.12]). A stable attractor $A$ is also called asymptotically stable. In this case $\varnothing \neq J^{+}(x) \subset A$ for every $x \in W^{+}(A)$ and the restricted flow in $W^{+}(A) \backslash A$ is particularly simple, since it is parallelizable with a compact global section (see $[3$, p. 83]). If $A$ is an attractor in $M$, it is well-known that the positive first prolongation

$$
D^{+}(A)=\bigcup_{x \in A} D^{+}(x)
$$

of $A$ is compact invariant and asymptotically stable with $W^{+}\left(D^{+}(A)\right)=$ $W^{+}(A)$ (see $\left[\mathbf{2}\right.$, Theorem 8.20]). Note that $D^{+}(A)=\left\{x \in M: L^{-}(x) \cap A \neq\right.$ $\varnothing\}$, if $A$ is an attractor.

Let $A \subset M$ be an isolated compact invariant set and let $\left(N, N^{+}, N^{-}\right)$be an isolating block of $A$. The final entrance time function $f: W^{+}(A) \backslash A \rightarrow \mathbb{R}$ 
defined by

$$
f(x)=\sup \{t \in \mathbb{R}: t x \in M \backslash N\}
$$

is lower semicontinuous. This follows immediately from the definition and the continuity of the flow. Obviously, $f(x) x \in \alpha^{+}$and $f(t x)=f(x)-t$ for every $t \in \mathbb{R}$.

Lemma 3.1. The final entrance time function $f$ is discontinuous at $x \in$ $W^{+}(A) \backslash A$ if and only if there are $x_{n} \rightarrow x$ such that $f\left(x_{n}\right) \rightarrow+\infty$.

Proof. If $f$ is discontinuous at $x$, there are $x_{n} \rightarrow x, x_{n} \in W^{+}(A) \backslash A$, and $\epsilon>0$ such that $f\left(x_{n}\right)>f(x)+\epsilon$, for every $n \in \mathbb{N}$. By the continuity of the flow and passing to a subsequence if necessary, there are $s_{n} \rightarrow f(x)$ such that $s_{n} x_{n} \in N^{+} \backslash N^{-}$, since $f(x) x \in \alpha^{+}$. Thus, eventually $f\left(x_{n}\right)>f(x)+\epsilon>s_{n}$. This means that $C^{+}\left(s_{n} x_{n}\right)$ exits $N$ eventually for all large enough $n \in \mathbb{N}$. The exit time function $\sigma^{+}: N \rightarrow[0,+\infty]$ defined by $\sigma^{+}(x)=0$, if $x \in N^{-}$, and

$$
\sigma^{+}(x)=\sup \left\{t>0:[0, t] x \in N \backslash N^{-}\right\},
$$

if $x \in N \backslash N^{-}$, is continuous. Hence, $f\left(x_{n}\right)>s_{n}+\sigma^{+}\left(s_{n} x_{n}\right) \rightarrow+\infty$, since $\sigma^{+}(f(x) x)=+\infty$. The converse is obvious.

Proposition 3.2. Let $A$ be an isolated invariant set of a flow $\phi$ on a separable, locally compact, metrizable space $M$ and let $\left(N, N^{+}, N^{-}\right)$be an isolating block of $A$. Then:

(i) $\phi$ maps $\mathbb{R} \times \operatorname{int}_{\partial N} \alpha^{+}$homeomorphically onto $\mathbb{R i n t}_{\partial N} \alpha^{+}$, and

(ii) $\phi$ maps $\mathbb{R} \times \partial_{\partial N} \alpha^{+}$homeomorphically onto $\mathbb{R} \partial_{\partial N} \alpha^{+}$.

Proof. It is clear that $\phi$ maps $\mathbb{R} \times \alpha^{+}$in a one-to-one manner onto $\mathbb{R} \alpha^{+}$. If now $f: W^{+}(A) \backslash A \rightarrow \mathbb{R}$ is the final entrance time function of the isolating block, then $f \mid \mathbb{R i n t}_{\partial N} \alpha^{+}$is continuous, by Lemmas 2.1 and 3.1. The inverse of $\phi \mid \mathbb{R} \alpha^{+}$is the map $h: \mathbb{R} \alpha^{+} \rightarrow \mathbb{R} \times \alpha^{+}$defined by $h(x)=(-f(x), f(x) x)$, which is therefore continuous on $\mathbb{R i n t}_{\partial N} \alpha^{+}$. To prove the second assertion, suppose that $\phi$ does not map $\mathbb{R} \times \partial_{\partial N} \alpha^{+}$homeomorphically onto $\mathbb{R} \partial_{\partial N} \alpha^{+}$. This means that there are $t, t_{n} \in \mathbb{R}$ and $x, x_{n} \in \partial_{\partial N} \alpha^{+}, n \in \mathbb{N}$, such that $t_{n} x_{n} \rightarrow t x$, but the sequence $\left\{\left(t_{n}, x_{n}\right): n \in \mathbb{N}\right\}$ does not converge to $(t, x)$. Suppose first that $\left\{t_{n}: n \in \mathbb{N}\right\}$ is bounded. If $s \in \mathbb{R}$ is any limit point of $\left\{t_{n}: n \in \mathbb{N}\right\}$ and $y \in \partial_{\partial N} \alpha^{+}$is any limit point of $\left\{x_{n}: n \in \mathbb{N}\right\}$, then $t x=s y$ and therefore $t=s$ and $x=y$. Since $\alpha^{+}$is compact, it follows that $t_{n} \rightarrow t$ and $x_{n} \rightarrow x$, contradiction. So $\left\{t_{n}: n \in \mathbb{N}\right\}$ is unbounded and passing to a subsequence if necessary we may assume that $t_{n} \rightarrow+\infty$. For every $s \in \mathbb{R}$ we have eventually $t_{n}-t+s>0$ for large values of $n \in \mathbb{N}$. So, $\left(t_{n}-t+s\right) x_{n} \in N$ and $\left(t_{n}-t+s\right) x_{n} \rightarrow s x$. Hence $s x \in N$ for every $s \in \mathbb{R}$, which contradicts the fact that $A$ is isolated.

Corollary 3.3. Let $\phi$ be a continuous flow on a separable, locally compact, metrizable space $M$ and let $A \subset M$ be an invariant continuum which is an 
isolated unstable attractor with region of attraction $W^{+}(A)$. Let $\left(N, N^{+}\right.$, $N^{-}$) be an isolating block of $A$ such that $N \subset W^{+}(A)$. Then,

$$
\mathbb{R} \partial_{\partial N} \alpha^{+}=\left\{x \in W^{+}(A) \backslash A: J^{+}(x) \nsubseteq \subseteq A\right\}
$$

and is homeomorphic image of $\mathbb{R} \times \partial_{\partial N} \alpha^{+}$under $\phi$.

Concerning the existence of explosions in the region of attraction of an isolated unstable attractor, we have the following:

Theorem 3.4. Let $A$ be an invariant continuum which is an isolated unstable attractor of a continuous flow on a connected, locally compact, metrizable space $M$. If $W^{+}(A) \backslash A$ is connected, then either $D^{+}(A)=M$ or there exists a point $x \in W^{+}(A) \backslash D^{+}(A)$ such that $\varnothing \neq J^{+}(x) \nsubseteq A$.

Proof. Suppose that the conclusion is not true. Since $D^{+}(A) \neq M$, we have $D^{+}(A) \neq W^{+}(A)$, because $A$ is an attractor and $M$ is assumed to be connected. The restricted flow in $W^{+}(A) \backslash D^{+}(A)$ is parallelizable and has a compact global section $S$. Let $\left(N, N^{+}, N^{-}\right)$be an isolating block of $A$ such that $N \subset D^{+}(A) \cup(0,+\infty) S$. By Lemma 3.1 and since we suppose that $\varnothing \neq J^{+}(x) \subset A$ for every $x \in W^{+}(A) \backslash D^{+}(A)$, the final entrance time function $f$ is continuous on $W^{+}(A) \backslash D^{+}(A)$ and hence bounded on $S$. If $W^{+}(A) \backslash D^{+}(A)$ is closed in $W^{+}(A) \backslash A$, then we arrive at a contradiction, since it is already open and $W^{+}(A) \backslash A$ is connected by assumption, which implies that $D^{+}(A)=A$, that is $A$ would be stable. So $W^{+}(A) \backslash D^{+}(A)$ is not closed in $W^{+}(A) \backslash A$, which means that there exists a sequence $\left\{x_{n}: n \in \mathbb{N}\right\}$ of points of $W^{+}(A) \backslash D^{+}(A)$ converging to some point $x \in D^{+}(A) \backslash A$. There are eventually $t_{n}<0$ such that $t_{n} x_{n} \in S$, for every $n \in \mathbb{N}$, and since $S$ is compact, we may assume that $t_{n} x_{n} \rightarrow y$ for some $y \in S$, passing to a subsequence. Obviously, $f\left(t_{n} x_{n}\right)=f\left(x_{n}\right)-t_{n}$. If $\left\{t_{n}: n \in \mathbb{N}\right\}$ had a bounded subsequence, and hence some convergent, we would have $y \in C(x)$, contradiction. So, we must have $t_{n} \rightarrow-\infty$. But then $x \in J^{+}(y)$, which contradicts our assumption in the beginning that the conclusion is not true.

The case $D^{+}(A)=M$ may occur as the example of the flow on $S^{1}$ with a single singular point shows. Moreover, in this example there is no explosion in $M \backslash A$. As an illustration of Theorem 3.4 consider the smooth flow on $\mathbb{R}^{2}$ defined by the differential equation (in polar coordinates)

$$
r^{\prime}=r(1-r) \text { and } \theta^{\prime}=\sin ^{2}\left(\frac{\theta}{2}\right) .
$$

Then, $\{(1,0)\}$ is an isolated unstable attractor with $W^{+}(1,0)=\mathbb{R}^{2} \backslash\{(0,0\}$ and $D^{+}(1,0)=S^{1}$. For $0<s<1$ or $s>1$ the orbit of the point $(s, 0) \in$ $W^{+}(1,0) \backslash D^{+}(1,0)$ explodes, because $J^{+}(s, 0)=S^{1}=D^{+}(1,0)$. By the way, the closed disc of radius $1 / 2$ centered at $(1,0)$ is an isolating block and the flow does not map $\mathbb{R} \times \alpha^{+}$homeomorphically onto $\mathbb{R} \alpha^{+}$. 
If $A$ is an isolated unstable attractor the question arises whether the flow explodes at orbits in $D^{+}(A) \backslash A$. In the example on $\mathbb{R}^{2}$ of differential Equation (1) we have $A=\{(1,0)\}, D^{+}(A)=J^{+}(1,0)=S^{1}$ and the disc $N$ of radius $1 / 2$ centered at $(1,0)$ is an isolating block. In this case the flow does not explode at the points of $D^{+}(A) \backslash A$, because $D^{+}(A) \cap \alpha^{+} \subset \operatorname{int}_{\partial N} \alpha^{+}$. On the other hand, there is a flow on the 2-torus with a single fixed point $x_{0}$, which is an isolated unstable attractor with $D^{+}\left(x_{0}\right)=T^{2}$ and the flow explodes at two orbits. This flow is induced on $T^{2}$ from the differential equation (in polar coordinates)

$$
r^{\prime}=(r-1)(2-r) \text { and } \theta^{\prime}=\sin ^{2}\left(\frac{\theta}{2}\right)
$$

on the annulus $1 \leq r \leq 2$ and $0 \leq \theta \leq 2 \pi$.

A sufficient condition for explosion in $D^{+}(A) \backslash A$ is given by the following:

Theorem 3.5. Let $A$ be an invariant continuum which is an isolated unstable attractor of a flow on a locally compact, metrizable space $M$. If the first integral Alexander-Spanier cohomology group $\bar{H}^{1}\left(W^{+}(A) ; \mathbb{Z}\right)$ is trivial, then there is a point $x \in D^{+}(A) \backslash A$ such that $J^{+}(x) \nsubseteq A$ and therefore the flow explodes at some orbit in $D^{+}(A) \backslash A$.

Proof. Suppose that $\varnothing \neq J^{+}(x) \subset A$ for every $x \in D^{+}(A) \backslash A$. Let $\chi: \mathbb{R} \rightarrow$ $(0,1)$ be an increasing homeomorphism. Let $\left(N, N^{+}, N^{-}\right)$be an isolating block of $A$ such that $N \subset W^{+}(A)$ and let $f: W^{+}(A) \backslash A \rightarrow \mathbb{R}$ be the final entrance time function. Let $g: D^{+}(A) \rightarrow S^{1}$ be the function defined by

$$
g(x)= \begin{cases}1, & \text { if } x \in A \\ \exp (2 \pi i \chi(f(x))), & \text { if } x \in D^{+}(A) \backslash A .\end{cases}
$$

Our assumption combined with Lemma 3.1 implies that $g$ is continuous. Since $D^{+}(A)$ is an asymptotically stable invariant continuum with $W^{+}\left(D^{+}(A)\right)=W^{+}(A)$, the restricted flow in $W^{+}(A) \backslash D^{+}(A)$ is parallelizable with a compact global section $S$. The set $W_{0}=D^{+}(A) \cup[0,+\infty) S$ is a positively invariant, compact, strong deformation retract of $W^{+}(A)$. If $W_{n}=D^{+}(A) \cup[n,+\infty) S, n \in \mathbb{N}$, then $W_{n}$ is a strong deformation retract of $W_{m}$ for $n \geq m$, and so the inclusion $W_{n} \subset W_{m}$ induces an isomorphism in Alexander-Spanier cohomology. Since

$$
D^{+}(A)=\bigcap_{n=0}^{\infty} W_{n},
$$

it follows from the continuity property of the Alexander-Spanier cohomology (see $\left[\mathbf{1 2}\right.$, p. 318]) that $\bar{H}^{1}\left(W^{+}(A) ; \mathbb{Z}\right) \cong \bar{H}^{1}\left(D^{+}(A) ; \mathbb{Z}\right)$, and so the latter is trivial. Recall that $\bar{H}^{1}\left(D^{+}(A) ; \mathbb{Z}\right)$ is naturally isomorphic to the abelian group of homotopy classes of continuous maps of $D^{+}(A)$ to $S^{1}[7$, Theorem 
8.1]. Thus, $g$ must be homotopic to a constant, that is $g=\exp (2 \pi i h)$, for some continuous function $h: D^{+}(A) \rightarrow \mathbb{R}$. Since $A$ is connected, $h$ takes an integer value, say $k$, on $A$. Moreover, for every $x \in D^{+}(A) \backslash A$, the function $\psi: C^{+}(x) \cup A \rightarrow \mathbb{Z}$ defined by $\psi(t x)=h(t x)-\chi(f(t x))$, for $t \geq 0$, and $\psi(A)=k$, is continuous and hence constant, because $\varnothing \neq L^{+}(x) \subset A$. This implies that $h(x)=\chi(f(x))+k$, for every $x \in D^{+}(A) \backslash A$. On the other hand, $L^{-}(x) \cap A \neq \varnothing$, for every $x \in D^{+}(A) \backslash A$, which means that there are $t_{n} \rightarrow-\infty$, such that the sequence $\left\{t_{n} x: n \in \mathbb{N}\right\}$ converges to some point of $A$. Consequently, $f\left(t_{n} x\right) \rightarrow+\infty$ and $k=h(A)=\lim _{n \rightarrow+\infty} h\left(t_{n} x\right)=1+k$, contradiction.

If $A$ is not isolated, Theorem 3.5 is not true. Consider for example the extension to the 2 -sphere $S^{2}$ of the parallel flow on $\mathbb{R}^{2}$. The point at infinity $\infty$ is fixed and is an unstable attractor, but not isolated. Its region of attraction is $W^{+}(\infty)=S^{2}=D^{+}(\infty)$ and $J^{+}(x)=\{\infty\}$ for every $x \in$ $S^{2} \backslash\{\infty\}$. A more interesting example is the following: In [1, Section 4] a continuous flow on the 4-sphere $S^{4}$ is constructed, in which the dyadic solenoid $Y=\left\{\left(z_{n}\right)_{n \geq 0}: z_{n} \in S^{1}\right.$ and $\left.z_{n}=z_{n+1}^{2}, n \geq 0\right\}$ embedds as an isolated minimal invariant set. Considering $S^{4}$ as the unreduced suspension of the 3 -sphere $S^{3}$, that is the quotient space obtained from $S^{3} \times[-1,1]$ by identifying $S^{3} \times\{ \pm 1\}$ to points, the poles of $S^{4}, Y$ embedds to $\{[y, 0]: y \in Y\}$ and the flow has the following properties:

(i) The poles of $S^{4}$ are fixed points.

(ii) If $y \notin Y$ and $s \neq \pm 1$, then $J^{+}[y, s]$ is the north pole and $J^{-}[y, s]$ is the south pole.

(iii) If $y \in Y$ and $0<s<1$, then $J^{+}[y, s]$ is the north pole and $L^{-}[y, s]=$ $Y$, while if $-1<s<0$, then $J^{-}[y, s]$ is the south pole and $L^{+}[y, s]=$ $Y$.

If $y_{0} \in Y$, the invariant continuum $A=Y \cup \overline{C\left[y_{0}, 1 / 2\right]}$ is a non-isolated unstable attractor with $D^{+}(A)=Y \cup \bigcup_{y \in Y} \overline{C[y, 1 / 2]}$ and $W^{+}(A)=S^{4} \backslash$ \{south pole\}. So $\bar{H}^{1}\left(W^{+}(A) ; \mathbb{Z}\right)$ is trivial but $J^{+}[y, s]=\{$ north pole $\} \subset A$ for every $[y, s] \in D^{+}(A) \backslash A$. In this example $\bar{H}^{1}(A ; \mathbb{Z}) \cong \bar{H}^{1}(Y ; \mathbb{Z})$ is also not finitely generated.

We shall examine now topological properties of an isolated unstable attractor $A$ in case there is essentially no explosion in $D^{+}(A) \backslash A$ and $D^{+}(A) \backslash A$ is not too bad.

Proposition 3.6. Let $A$ be an invariant continuum which is an isolated unstable attractor of a flow on a locally compact, metrizable space $M$. If $D^{+}(A) \backslash A$ is locally connected, then it has a finite number of connected components. If moreover $\varnothing \neq J^{+}(x) \subset A$ for every $x \in D^{+}(A) \backslash A$, then the number of the connected components of $D^{+}(A) \backslash A$ is not greater than $\operatorname{rank} \bar{H}^{1}\left(W^{+}(A) ; \mathbb{Z}\right)$. 
Proof. We shall prove first that $D^{+}(A) \backslash A$ has a finite number of connected components. Every connected component of $D^{+}(A) \backslash A$ is open in $D^{+}(A)$, since $D^{+}(A) \backslash A$ is assumed to be locally connected. Suppose that $D^{+}(A) \backslash A$ has an infinite number of connected components $C_{n}, n \in \mathbb{N}$. Let $\left(N, N^{+}, N^{-}\right)$be an isolating block of $A$. Then, $C_{n} \cap \partial N \neq \varnothing$ and if we pick points $x_{n} \in C_{n} \cap \partial N, n \in \mathbb{N}$, they have some limit point $x \in D^{+}(A) \cap \partial N \subset D^{+}(A) \backslash A$, which must threfore be in some connected component $C$ of $D^{+}(A) \backslash A$. But then $C$ is an open neighbouhood of $x$ in $D^{+}(A)$ and so we must have $x_{n} \in C$ for infinitely many values of $n$, contradiction. Thus, $D^{+}(A) \backslash A$ has a finite number of connected components, say $C_{1}, \ldots, C_{k}$. To prove the second assertion, note first that $C_{j} \cup A$ is an invariant continuum for all $j=1,2, \ldots, k$. Let $f: W^{+}(A) \backslash A \rightarrow \mathbb{R}$ be the final entrance time function of the isolating block. For each $j=1,2, \ldots, k$ let $g_{j}: D^{+}(A) \rightarrow S^{1}$ be the function defined by

$$
g_{j}(x)= \begin{cases}1, & \text { if } x \in D^{+}(A) \backslash C_{j}, \\ \exp (2 \pi i \chi(f(x))), & \text { if } x \in C_{j},\end{cases}
$$

where $\chi: \mathbb{R} \rightarrow(0,1)$ is an increasing homeomorphism. Since $D^{+}(A) \backslash A$ has finitely many connected components and $f$ is continuous on $D^{+}(A) \backslash$ $A, g_{j}$ is continuous. The argument in the proof of Theorem 3.5 shows that $g_{j} \mid C_{j} \cup A$ is not homotopic to a constant. Consequently, $\left(g_{j} \mid C_{j} \cup\right.$ $A)^{m}$, for $m \neq 0$, defines a nonzero element of $\bar{H}^{1}\left(C_{j} \cup A ; \mathbb{Z}\right)$. We shall prove that $g_{1}, \ldots, g_{k}$ define a set of linearly independent cohomology classes in $\bar{H}^{1}\left(D^{+}(A) ; \mathbb{Z}\right)$. Indeed, let $n_{1}, \ldots, n_{k} \in \mathbb{Z}$ be such that $g_{1}^{n_{1}} \ldots g_{k}^{n_{k}}$ is homotopic to a constant. There is a continuous function $h: D^{+}(A) \rightarrow$ $\mathbb{R}$ such that $g_{1}^{n_{1}} \ldots g_{k}^{n_{k}}=\exp (2 \pi i h)$. It follows from the definitions that $\left(g_{j} \mid C_{j} \cup A\right)^{n_{j}}=\exp \left(2 \pi i\left(h \mid C_{j} \cup A\right)\right)$, and so it is homotopic to a constant. Hence $n_{j}=0$, for every $j=1,2, \ldots, k$. This completes the proof.

Theorem 3.7. Let $A$ be an invariant continuum and an isolated unstable attractor of a flow $\phi$ on a locally compact ANR $M$, such that:

(i) $D^{+}(A) \backslash A$ is an ANR, and

(ii) $\varnothing \neq J^{+}(x) \subset A$ for every $x \in D^{+}(A) \backslash A$.

Then $\bar{H}^{q}(A ; \mathbb{Z})$ is finitely generated for all $q$.

Proof. We observe first that the restricted flow on the invariant locally compact subspace $D^{+}(A) \backslash A$ of $M$ is parallelizable with a compact global section. Actually, if $\left(N, N^{+}, N^{-}\right)$is an isolating block of $A$, such that $N \subset W^{+}(A)$, then $S=D^{+}(A) \cap \alpha^{+} \subset \operatorname{int}_{\partial N} \alpha^{+}$, by Assumption (ii) and Lemma 2.1, and is a compact global section to the restricted flow on $D^{+}(A) \backslash A$, by Proposition 3.2. The assumption that $D^{+}(A) \backslash A$ is an ANR implies that $S$ is an ANR also. It follows that $H^{q}(S ; \mathbb{Z})$ is finitely generated for all $q$ and we 
have the chain of isomorphisms

$$
\begin{aligned}
\bar{H}^{q+1}\left(D^{+}(A), A ; \mathbb{Z}\right) & \cong \bar{H}_{c}^{q+1}\left(D^{+}(A) \backslash A ; \mathbb{Z}\right) \cong \bar{H}_{c}^{q+1}(\mathbb{R} \times S ; \mathbb{Z}) \\
& \cong \bar{H}^{q}(S ; \mathbb{Z}) \cong H^{q}(S ; \mathbb{Z}),
\end{aligned}
$$

where $\bar{H}_{c}^{*}$ denotes Alexander-Spanier cohomology with compact supports (see $\left[\mathbf{1 2}\right.$, p. 320]). From the exact sequence of the pair $\left(D^{+}(A), A\right)$ we deduce that $\bar{H}^{q}(A ; \mathbb{Z})$ is finitely generated for all $q$.

\section{Singular unstable attractors in the 2-sphere.}

Let $\phi$ be a continuous flow on a connected 2-manifold $M$ having a fixed point $x_{0}$, which we assume to be an isolated invariant set. Every neighbourhood of $x_{0}$ contains an isolating compact neighbourhood of $x_{0}$, which is a compact 2manifold with boundary. The interior $V$ of such a neighbourhood is an open 2-manifold of finite genus and with finitely many ends. We can reparametrize the local flow in $V$ to get a flow in $V$ with respect to which $\left\{x_{0}\right\}$ is the only invariant set in $V$, apart $V$ itself. It follows from the Smoothing Theorem of C. Gutierrez (see [10]) that the flow in $V$ is topologically equivalent to a smooth flow, that is there exists a homeomorphism $h: V \rightarrow V$ which maps the oriented orbits in $V$ onto the oriented orbits of some smooth flow in $V$. Since $\left\{x_{0}\right\}$ is an isolated invariant set with respect to the smooth flow also, it has a smooth isolating block $\left(P, P^{+}, P^{-}\right)$with respect to the smooth flow. Clearly, $\left(h^{-1}(P), h^{-1}\left(P^{+}\right), h^{-1}\left(P^{-}\right)\right)$is an isolating block for $\left\{x_{0}\right\}$ with respect to the initial flow $\phi$, and $h^{-1}(P)$ is a topological compact 2manifold with boundary $h^{-1}\left(P^{+}\right) \cup h^{-1}\left(P^{-}\right)$. The sets $h^{-1}\left(P^{+}\right), h^{-1}\left(P^{-}\right)$ are topological compact 1-manifolds with common boundary $h^{-1}\left(P^{+}\right) \cap$ $h^{-1}\left(P^{-}\right)$, which is a finite set. If we start with a disc neighbourhood, then $V$ has genus zero and so does $h^{-1}(P)$. Summarizing we have the following:

Lemma 4.1. Let $\phi$ be a continuous flow on a connected 2-manifold $M$ and let $x_{0} \in M$ be a fixed point of the flow which is an isolated invariant set. Then every neighbourhood of $x_{0}$ contains an isolating block $\left(N, N^{+}, N^{-}\right)$ such that $N$ is a genus zero compact 2-manifold with boundary, and $N^{+}$, $N^{-}$are compact 1-manifolds with common boundary $N^{+} \cap N^{-}$, which is a finite set.

Proposition 4.2. The set $\partial_{\partial N} \alpha^{+}$is finite and $\alpha^{+}$has finitely many connected components.

Proof. Suppose on the contrary that $\partial_{\partial N} \alpha^{+}$has infinitely many points. By compactness, there is a sequence $\left\{x_{n}: n \in \mathbb{N}\right\}$ in $\partial_{\partial N} \alpha^{+}$converging to a point $x \in \partial_{\partial N} \alpha^{+}$. Let $I$ be a small open interval in $N^{+} \backslash N^{+} \cap N^{-}$ containing $x$. Passing to a subsequence if necessary, we may assume that $x_{n} \in I$ and if $\left[x_{n}, x\right]$ is the subinterval of $I$ with endpoints $x_{n}$ and $x$, then 
$\left[x_{n+1}, x\right] \subset\left[x_{n}, x\right]$, for every $n \in \mathbb{N}$. Let $U$ be a disc neighbourhood of $x_{0}$ contained in $\operatorname{int} N$. There is a $T>0$ such that $C^{+}(T x) \subset U$. A similar argument as in the proof of Lemma 2.1(a) shows that we must also have $C^{+}\left(T x_{n}\right) \subset U$, for large $n$. Since the flow is transverse to $N^{+} \backslash N^{+} \cap N^{-}$ into $N$, some small closed interval $J \subset I$ containing $x$ in its interior is a local section to the flow of extent $T$, by [11, Lemma VII.2.9]. This means that the flow maps $[-T, T] \times J$ homeomorphically onto its image. So, $\phi([0, T] \times J)$ is a disc in $N$ and $J=\phi([0, T] \times J) \cap \partial N$. For large $n$ we have $x_{n} \in J$. The simple closed curve $T\left[x_{n}, x\right] \cup C^{+}\left(T x_{n}\right) \cup C^{+}(T x) \cup\left\{x_{0}\right\}$ bounds a disc $V_{n}$ in $U$, by the Jordan-Schönflies theorem. It follows that the set $D_{n}=\phi([0, T] \times J) \cup V_{n}$ is a disc in $N$ and $\partial D_{n}=\left[x_{n}, x\right] \cup C^{+}\left(x_{n}\right) \cup C^{+}(x) \cup\left\{x_{0}\right\}$. It is evident that $D_{n}$ is positively invariant, since the flow is transverse to $\left[x_{n}, x\right]$ into $D_{n}$ and no positive semiorbit starting on $\left(x_{n}, x\right) \cup \operatorname{int} D_{n}$ can cross $\partial D_{n}$. This implies that $\left[x_{n}, x\right] \subset \alpha^{+}$and hence $x_{n+1} \in \operatorname{int}_{\partial N} \alpha^{+}$. This contradiction proves the first assertion. The second follows from the first.

In Proposition 4.2 we have not assumed that the fixed point is an attractor. Combining it now with Corollary 3.3 we get the following:

Corollary 4.3. Let $x_{0}$ be a fixed point of a continuous flow on a connected 2-manifold $M$. If $x_{0}$ is an isolated unstable attractor, then the flow on $W^{+}\left(x_{0}\right) \backslash\left\{x_{0}\right\}$ explodes at finitely many orbits.

In the rest of this section we shall be concerned with the structure of the flow in the region of attraction of a fixed point of a flow on the 2sphere $S^{2}$, which is an unstable attractor. We are mainly interested in the topological structure of the positive first prolongation of the fixed point and the dynamics of the flow in it. Most of the results will be proved without the assumption that the fixed point is an isolated invariant set.

Proposition 4.4. Let $x_{0} \in S^{2}$ be a fixed point of a continuous flow $\phi$ on $S^{2}$. If $x_{0}$ is an unstable attractor, then $L^{+}(x)=L^{-}(x)=\left\{x_{0}\right\}$ for every $x \in D^{+}\left(x_{0}\right)$.

Proof. Since $D^{+}\left(x_{0}\right)$ is a compact invariant set, we have $\varnothing \neq L^{-}(x) \subset$ $D^{+}\left(x_{0}\right)$ for every $x \in D^{+}\left(x_{0}\right)$. If $y \in L^{-}(x)$, then $x_{0} \in L^{+}(y) \subset L^{-}(x)$. Suppose that $L^{-}(x)$ contains and some other point $z \neq x_{0}$. Then, $z \in$ $D^{+}\left(x_{0}\right)$ and $z$ is not fixed. Moreover, $L^{+}(z)=L^{-}(z)=\left\{x_{0}\right\}$, because $x_{0}$ is the only fixed point in $W^{+}\left(x_{0}\right)$ (see [11, Proposition 1.11]). Thus, $\overline{C(z)}$ is a simple closed curve and $S^{2} \backslash \overline{C(z)}$ has two connected components with common boundary $\overline{C(z)}$, whose closures are closed discs by the JordanSchönflies theorem. Both are invariant and $C(x)$ is contained in one of them, call it $B$. Let $S$ be a local section to the flow at $z$. That is, $S$ is an open arc passing through $z$ and $\phi$ maps $(-\epsilon, \epsilon) \times S$ homeomorphically onto an open neighbourhood of $z$ for some $\epsilon>0$ (see [11, Corollary 2.6]). Since $z \in L^{-}(x)$, there is some $t<0$ such that $t x \in S$ and we can choose $t$ 
such that $C^{+}(t x) \cap S=\{t x\}$, because $L^{+}(x)=\left\{x_{0}\right\}$. Let $J$ be the open subarc in $S$ with endpoints $t x$ and $z$. The set $K=\overline{C^{+}(t x)} \cup J \cup \overline{C^{+}(z)}$ is a simple closed curve and $S^{2} \backslash K$ has two connected components. One of them is positively invariant and the other is negatively invariant. Let $D$ be the one that is negatively invariant. Then $(-\infty, 0) z \subset D$. Since $z \in L^{-}(x)$ and $J \subset B$, there is some $s<t$ such that $s x \in J$. But then $t x \in(0,+\infty)(s x) \subset S^{2} \backslash \bar{D} \subset S^{2} \backslash \bar{J}$, contradiction.

Theorem 4.5. Let $x_{0}$ be a fixed point of a continuous flow $\phi$ on $S^{2}$. If $x_{0}$ is an unstable attractor, then every point $x \in D^{+}\left(x_{0}\right) \backslash\left\{x_{0}\right\}$ has an open neighbourhood $V$ such that $\left(D^{+}\left(x_{0}\right) \backslash\left\{x_{0}\right\}\right) \cap V$ is homeomorphic to the open interval $(-1,1)$ or the open rectangle $(-1,1) \times(-1,1)$ or to $[-1,1) \times(-1,1)$.

Proof. Let $x \in D^{+}\left(x_{0}\right) \backslash\left\{x_{0}\right\}$. Since $x$ is not fixed, there is a local section to the flow through $x$. Thus there are an open arc $S$ containing $x$ and $\epsilon>0$ such that $\phi$ maps $(-\epsilon, \epsilon) \times S$ homeomorphically onto an open neighbourhood of $x$. We can shrink $S$, if necessary, so that $C(x) \cap S=\{x\}$, because $L^{+}(x)=L^{-}(x)=\left\{x_{0}\right\}$, by Proposition 4.4. The set $S \backslash\{x\}$ is the disjoint union of two open intervals $S_{1}, S_{2}$ in $S$ with one common endpoint $x$. If there are open intervals $I \subset S_{1}$ and $J \subset S_{2}$ with common endpoint $x$ such that $I \cup J \subset W^{+}\left(x_{0}\right) \backslash D^{+}\left(x_{0}\right)$, or $I \cup J \subset D^{+}\left(x_{0}\right)$, or $I \subset W^{+}\left(x_{0}\right) \backslash D^{+}\left(x_{0}\right)$ and $J \subset D^{+}\left(x_{0}\right)$, or vice versa, then we are done. We shall prove that otherwise we are led to a contadiction. Suppose that every open interval $I \subset S_{1}$ with one endpoint $x$ contains points of $D^{+}\left(x_{0}\right)$ and $W^{+}\left(x_{0}\right) \backslash D^{+}\left(x_{0}\right)$. There is a sequence $\left\{x_{n}: n \in \mathbb{N}\right\}$ of points in $D^{+}\left(x_{0}\right) \cap S_{1}$, which converges monotonically to $x$, such that $\left[x_{n}, x_{n+1}\right] \cap\left(W^{+}\left(x_{0}\right) \backslash D^{+}\left(x_{0}\right)\right) \neq \varnothing$, where $\left[x_{n}, x_{n+1}\right]$ denotes the closed interval on $S_{1}$ with endpoints $x_{n}$ and $x_{n+1}$. The monotonicity of convergence to $x$ means that $\left[x, x_{n+1}\right] \subset\left[x, x_{n}\right]$ for every $n \in \mathbb{N}$. From Proposition 4.4 we have $L^{+}\left(x_{n}\right)=L^{-}\left(x_{n}\right)=\left\{x_{0}\right\}$ for every $n \in \mathbb{N}$. Let $D$ be the closed invariant disc in $S^{2}$ bounded by the simple closed curve $\overline{C(x)}$, such that $\left[x, x_{n}\right] \subset D$ for some (and hence for all) $n \in \mathbb{N}$. Then, $\overline{C\left(x_{n}\right)}$ bounds a closed disc $D_{n} \subset D$ such that $\left[x, x_{n}\right] \subset D \backslash \operatorname{int} D_{n}$. The monotonicity implies that $D_{n} \subset D_{m}$ and $\left[x_{n}, x_{m}\right] \subset D_{m} \backslash \operatorname{int} D_{n}$ for $n<m$. If $y_{n} \in\left[x_{n}, x_{n+1}\right] \cap\left(W^{+}\left(x_{0}\right) \backslash D^{+}\left(x_{0}\right)\right)$, then $C\left(y_{n}\right) \subset \operatorname{int} D_{n+1} \backslash D_{n}$ and it hits some connected component of a global section $\Sigma$ of the parallelizable restricted flow in $W^{+}\left(x_{0}\right) \backslash D^{+}\left(x_{0}\right)$, which is a compact 1-manifold, that is $\Sigma$ is finite union of disjoint simple closed curves $\Sigma_{1}, \ldots, \Sigma_{k}$. If $C\left(y_{n}\right) \cap \Sigma_{j} \neq \varnothing$, then $\Sigma_{j} \subset \operatorname{int} D_{n+1} \backslash D_{n}$, because $\Sigma \cap \overline{C\left(x_{n+1}\right) \cup C\left(x_{n}\right)}=\varnothing$. But the sets $\operatorname{int} D_{n+1} \backslash D_{n}, n \in \mathbb{N}$ are disjoint and infinitely many. This contradiction completes the proof.

From Proposition 3.6 and Theorem 4.5 we have the following:

Corollary 4.6. If $x_{0} \in S^{2}$ is a fixed point of a continuous flow on $S^{2}$ and is an isolated unstable attractor, then $D^{+}\left(x_{0}\right) \backslash\left\{x_{0}\right\}$ is an ANR and has 
finitely many connected components. Each connected component is either an orbit homeomorphic to $\mathbb{R}$ or a noncompact 2-manifold with nonempty and noncompact boundary, with finitely many boundary components.

The dynamics in the positive first prolongation of a fixed point of a continuous flow on $S^{2}$ which is an unstable attractor is described by the following:

Theorem 4.7. If $x_{0} \in S^{2}$ is a fixed point of a continuous flow on $S^{2}$ and is an unstable attractor, then the restricted flow in $D^{+}\left(x_{0}\right) \backslash\left\{x_{0}\right\}$ is completely unstable.

Proof. Suppose on the contrary that there is some $x \in D^{+}\left(x_{0}\right) \backslash\left\{x_{0}\right\}$, which is non-wandering with respect to the restricted flow in $D^{+}\left(x_{0}\right) \backslash\left\{x_{0}\right\}$. This means that there are a sequence $\left\{x_{n}: n \in \mathbb{N}\right\}$ of points of $D^{+}\left(x_{0}\right) \backslash\left\{x_{0}\right\}$ and $t_{n} \rightarrow+\infty$ such that $x_{n} \rightarrow x$ and $t_{n} x_{n} \rightarrow x$. By Proposition $4.4, \overline{C(x)}$ is a simple closed curve and bounds an invariant closed disc $D$ in $S^{2}$ such that $C\left(x_{n}\right) \subset \operatorname{int} D$ for every $n \in \mathbb{N}$, passing to a subsequence if necessary. Let $S$ be an open arc that is a local section to the flow at $x$ of some extent $\epsilon>0$, and such that $C(x) \cap S=\{x\}$. Let $I$ be the connected component of $S \backslash\{x\}$ which lies in $\operatorname{int} D$. For large enough values of $n$, there are $\left|s_{n}\right|<\epsilon$ and $\left|r_{n}\right|<\epsilon$ such that $s_{n} x_{n} \in I$ and $\left(t_{n}+r_{n}\right) x_{n} \in I$. Since $t_{n} \rightarrow+\infty$, eventually we have $t_{n}+r_{n}>s_{n}+\epsilon$. Let $T_{n}>\epsilon$ be the first time $C^{+}\left(s_{n} x_{n}\right)$ hits $I$. If $\left[s_{n} x_{n}, T_{n} x_{n}\right]$ denotes the closed interval in $I$ with endpoints $s_{n} x_{n}$ and $T_{n} x_{n}$, then $K=\left[s_{n}, T_{n}\right] x_{n} \cup\left[s_{n} x_{n}, T_{n} x_{n}\right]$ is a simple closed curve and bounds a closed disc $D^{\prime} \subset \operatorname{int} D$, which is positively or negatively invariant. But then $L^{-}\left(x_{n}\right) \subset D^{\prime}$ or $L^{+}\left(x_{n}\right) \subset D^{\prime}$, which contradicts that $x_{n} \in D^{+}\left(x_{0}\right) \backslash\left\{x_{0}\right\}$.

Recall that a separatrix in a completely unstable flow is an orbit with nonempty positive prolongational limit set. It follows from Corollary 4.3 that if $x_{0}$ is a fixed point of a continuous flow on $S^{2}$ which is an isolated unstable attractor, then the restricted flow in $D^{+}\left(x_{0}\right) \backslash\left\{x_{0}\right\}$ is completely unstable with finitely many separatrices.

\section{References}

[1] G. Allaud and E.S. Thomas, Almost periodic minimal sets, J. Differential Equations, 15 (1974), 158-171, MR 49 \#4049, Zbl 0299.58010.

[2] N.P. Bhatia and O. Hajek, Local Semi-Dynamical Systems, Lecture Notes in Math., 90, Springer-Verlag, Berlin/New York, 1969, MR 40 \#4559, Zbl 0176.39102.

[3] N.P. Bhatia and G.P. Szegö, Stability Theory of Dynamical Systems, Grundl. der Math. Wissenschaften, 161, Springer-Verlag, Berlin/New York, 1970, MR 44 \#7077, Zbl 0213.10904.

[4] R. Churchill, Isolated invariant sets in compact metric spaces, J. Differential Equations, 12 (1972), 330-352, MR 49 \#1536, Zbl 0238.54044.

[5] C.C. Conley, Isolated Invariant Sets and the Morse Index, Conf. Board Math. Sci., 38, American Math. Soc., Providence, RI, 1978, MR 80c:58009, Zbl 0397.34056. 
[6] C.C. Conley and R. Easton, Isolated invariant sets and isolating blocks, Trans. Amer. Math. Soc., 158 (1971), 35-61, MR 43 \#5551, Zbl 0223.58011.

[7] C.H. Dowker, Mapping theorems for noncompact spaces, Amer. J. Math., 69 (1947), 200-247, MR 8,594g, Zbl 0037.10101.

[8] B. Günther, Construction of differentiable flows with prescribed attractor, Topology Appl., 62 (1995), 87-91, MR 96e:58095, Zbl 0822.54015.

[9] B. Günther and J. Segal, Every attractor of a flow on a manifold has the shape of a finite polyhedron, Proc. Amer. Math. Soc., 119 (1993), 321-329, MR 93k:54044, Zbl 0822.54014.

[10] C. Gutierrez, Smoothing continuous flows on two manifolds and recurrences, Ergodic Theory Dynamical Systems, 6 (1986), 17-44, MR 87k:58222, Zbl 0606.58042.

[11] O. Hajek, Dynamical Systems in the Plane, Academic Press, New York, 1968, MR 39 \#1767, Zbl 0169.54401.

[12] E. Spanier, Algebraic Topology, Mc Graw-Hill, New York, 1966, MR 35 \#1007, Zbl 0145.43303.

Received July 21, 2000 and revised May 21, 2001.

Department of Mathematics

University of CRETE

GR-71409 IRAKLION

Greece

E-mail address: athanako@math.uoc.gr 